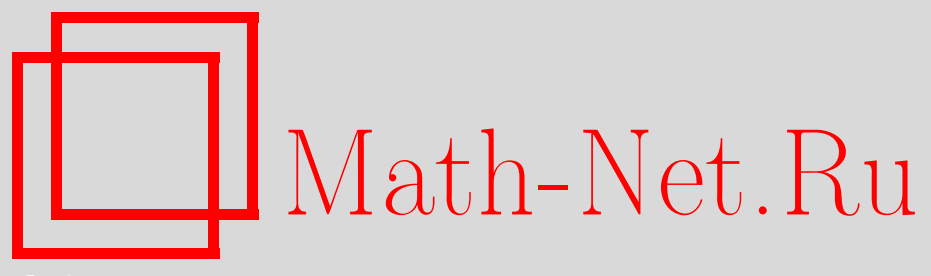

П. Г. Гриневич, А. Е. Миронов, С. П. Новиков, Двумерный оператор Шрёдингера: эволюционные $(2+1)$ системы и их новые редукции; двумерная иерархия Бюргерса и данные обратной задачи, УМH, 2010, том 65, выпуск 3, 195-196

DOI: https://doi.org/10.4213/rm9363

Использование Общероссийского математического портала Math-Net.Ru подразумевает, что вы прочитали и согласны с пользовательским соглашением http://www . mathnet.ru/rus/agreement

Параметры загрузки:

IP: 34.239 .49 .27

26 апреля 2023 г., 05:09:59

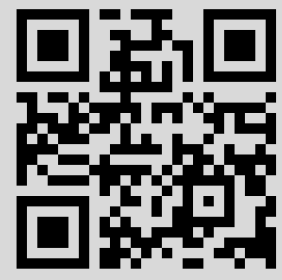




\title{
Двумерный оператор Шрёдингера: \\ эволюционные (2+1)-системы и их новые редукции; двумерная иерархия Бюргерса и данные обратной задачи
}

\author{
П. Г. Гриневич, А. Е. Миронов, С. П. Новиков
}

Теория $(2+1)$-систем, базирующихся на двумерном операторе Шрёдингера, была начата в 1976 г. в [1], [2]. Аналог пар Лакса [1] имеет вид $\dot{L}=[H, L]-f L$, где $L=\partial_{x} \partial_{y}+G \partial_{y}+S$, а $H, f$ - некоторые дифференциальные операторы. В [2] были построены алгебро-геометрические решения таких систем, а следовательно, и их полные иерархии. В [2] была начата теория обратных задач для эллиптического оператора $L(x \rightarrow z, y \rightarrow \bar{z})$, где данные снимаются с комплексной кривой Ферми нулевого уровня $L \Psi=0$. Ряд интересных систем были найдены в [3]. Однако первая система такого рода, построенная в [1], не изучалась до работы [4], в которой были найдены преобразовния Бэклунда и была указана редукция типа Бюргерса ${ }^{1}$. Авторами настоящей работы недавно были найдены приложения данной системы, предложены самосопряженная иерархия и двумерная иерархия Бюргерса, а также найдены редукции на данные обратной задачи для важных физических операторов (“чисто магнитный оператор Паули"). Мы обозначим эту систему GKMMN по фамилиям авторов, исследовавших ее. Пусть $H=\partial_{x}^{2}+\partial_{y}^{2}+F \partial_{y}+A, L=\partial_{x} \partial_{y}+G \partial_{y}+S$ (их можно привести к такому виду калибровочным преобразованием).

ПредлоЖЕниЕ. Система GKMMN имеет вид:

$$
\begin{gathered}
G_{t}=\square G+\left(F^{2} / 4\right)_{x}-\left(G^{2}\right)_{x}-A_{x}+2 S_{y}, \quad S_{t}=\square S-2(G S)_{x}+(F S)_{y}, \\
F_{x}=2 G_{y}, \quad A_{y}=2 S_{x}, \quad f=2 G_{x}-F_{y}, \quad \square=\partial_{x}^{2}-\partial_{y}^{2} .
\end{gathered}
$$

СледСтвие 1. Система GKMMN допускает вещественную редукцию.

Следствие 2. Система GKMMN обладает редукцией $S=0$ ("2D-уравнение Бюргерса" $\left.B_{2}\right)$. Полная иерархия строится с помощью теоремы 2.

СледСтвиЕ 3. Для системы GKMMN и ее стационарной задачи оператор $H$ может быть самосопряженным только в тривиальных случаях. Для $H=\partial_{x}^{2}+\partial_{y}^{2}+$ $F \partial_{y}+A$ в самосопряженном случае магнитное поле $F_{x} / i$ и электрический потенииал $A-F^{2} / 4-F_{y} / 2$ вещественны.

При наложенных условиях система GKMMN становится сильно переопределенной, приводя к полному вырождению.

ГиПотезА. Для гладкого периодического самосопряженного 2D-оператора L второго порядка полное многообразие $W(L)$ комплексных функиий Блоха-Флоке за исключением тривиальных случаев (сводящихся к одной переменной) не содержит открытых в топологии Зариского частей $\Gamma \subset W(L)$ алгебрачческих кривых кроме уровней $\varepsilon=$ const, найденных в [2].

Подстановка $G=-(\log c)_{x}, F=-2(\log c)_{y}, A=-2 u_{x}, S=-u_{y}$ приводит систему к виду: $\left[\left(c_{t}-\square c\right) c^{-1}\right]_{x}=-2 \square u,\left(u_{t}+\square u\right)_{y}=2\left(u_{y} c_{x} c^{-1}\right)_{x}-2\left(u_{y} c_{y} c^{-1}\right)_{y}$. При этом

П. Гриневич поддержан РФФИ (грант № 09-01-12148-офи-м), грантом НШ-5413.2010.1 и программой Президиума РАН “Фундаментальные проблемы нелинейной динамики”, А. Миронов - интеграционным проектом СО РАН (грант 65) и грантом НШ-5770.2010.1, С. Новиков РФФИ (грант № 08-01-00054-а).

${ }^{1} \mathrm{~K}$ сожалению, в [4] эта система была представлена как новая, хотя работа [1] была включена в список литературы [4]. Работа [2], в которой в 1976 г. были найдены алгебро-геометрические решения этих систем, в [4] вообще не цитируется. Это создает ложное впечатление, что до 1980 г. никакого прогресса не было, заменяя хронологический порядок участия авторов на противоположный. 
$B_{2}$-редукция, отвечающая $S=0$, имеет вид: $c_{t}-\square c=(U(x, t)+V(y, t)) c$, где $U, V-$ произвольные функции.

Алгебро-геометрические данные обратной задачи таковы: дана риманова поверхность $\Gamma$ рода $g$ с двумя точками $\infty_{1}, \infty_{2}$ и локальными параметрами $k_{1}^{-1}, k_{2}^{-1}$, где $k_{j}^{-1}\left(\infty_{j}\right)=0$, а также дивизор полюсов $\mathscr{D}=\mathscr{P}_{1}+\cdots+\mathscr{P}_{g}$. Двухточечная функция Бейкера-Ахиезера $\Psi(x, y, \mathscr{P}), \mathscr{P} \in \Gamma$, мероморфна на $\Gamma \backslash\left(\infty_{1} \cup \infty_{2}\right)$, имеет простые полюсы в точках $\mathscr{P}_{1}, \ldots, \mathscr{P}_{g}$ и асимптотики около $\infty_{j}$ :

$$
\Psi=e^{k_{1} x+k_{1}^{2} t}\left(1+u k_{1}^{-1}+O\left(k_{1}^{-2}\right)\right), \quad \Psi=c e^{k_{2} y+k_{2}^{2} t}\left(1+v k_{2}^{-1}+O\left(k_{2}^{-2}\right)\right) .
$$

Функция $\Psi$ удовлетворяет уравнению $L \Psi=0$ и уравнениям (1), с параметрами $(c, u)$. Вещественная редукиия (1) такова: имеется антиголоморфная инволюция $\sigma$ такая, что $\sigma\left(P_{j}\right)=P_{j}, \sigma^{*}\left(k_{j}\right)=-\bar{k}_{j}, \sigma(D)=D$.

Теорема 1. Возъмем риманову поверхность $\Gamma=\Gamma^{\prime} \cup \Gamma^{\prime \prime}$ (приводимую), әде $\Gamma^{\prime} \cap$ $\Gamma^{\prime \prime}=Q_{0} \cup \cdots \cup Q_{k}, \infty_{1} \in \Gamma^{\prime}, \infty_{2} \in \Gamma^{\prime \prime}, g^{\prime}-$ род $\Gamma^{\prime}, g^{\prime \prime}-$ род $\Gamma^{\prime \prime}$. Фиксируем дивизор полюсов первого порлдка $\mathscr{D}^{\prime} \cup \mathscr{D}^{\prime \prime},\left|\mathscr{D}^{\prime}\right|=g^{\prime}+k,\left|\mathscr{D}^{\prime \prime}\right|=g^{\prime \prime}, \mathscr{D}^{\prime} \in \Gamma^{\prime}, \mathscr{D}^{\prime \prime} \in \Gamma^{\prime \prime} ;$ пусть все точки $\infty_{j}, \mathscr{D}^{\prime}, \mathscr{D}^{\prime \prime}, Q$ различны. Строим функиию $\Psi$ вида: $\Psi=\Psi^{\prime \prime}$ на $\Gamma^{\prime \prime}-$ обычная одноточечная функиия Бейкера-Ахиезера с дивизором $\mathscr{D}^{\prime \prime}, \Psi^{\prime \prime}=e^{k_{2} y+k_{2}^{2} t} \times$ $\left(1+u k_{2}^{-1}+\cdots\right) ; \Psi=\Psi^{\prime}$ на $\Gamma^{\prime}, \Psi^{\prime}=c e^{k_{1} x+k_{1}^{2} t}\left(1+v k_{1}^{-1}+\cdots\right)$ с дивизором $\mathscr{D}^{\prime}$ степени $g^{\prime}+k$ и условиями $\left.\Psi^{\prime}\right|_{Q_{l}}=\left.\Psi^{\prime \prime}\right|_{Q_{l}}, l=0, \ldots, k$. Тогда $L \Psi=0,(\dot{L}-[H, L]) \Psi=0$, где $S=0\left(\right.$ m.e. $\left.u_{y}=0\right)$.

Возникающую иерархию систем с высшими временами при условии $S=0$ мы называем "2D-иерархией Бюргерса" $B_{2}$. Имеются два случая: 1$) x, y \in \mathbb{R}\left(B_{2}-\mathrm{I}\right)$; 2) $x \rightarrow z, y \rightarrow \bar{z}, \partial_{x} \rightarrow \partial_{z}, \partial_{y} \rightarrow \partial_{\bar{z}}\left(B_{2}\right.$-II $)$.

Теорема 2. Для переменных $z, \bar{z}$ редукиия системы GKMMN к классу самосопряженных операторов $L=\partial_{z} \partial_{\bar{z}}+G \partial_{\bar{z}}+S, 2 G_{\bar{z}}=F_{z} \in \mathbb{R}, S \in \mathbb{R}$, совместна с динамикой по времени it:

$$
\left[\left(c_{t}-4 c_{x y}\right) c^{-1}\right]_{z}=8 u_{x y}, \quad\left(u_{t}+4 u_{x y}\right)_{\bar{z}}=2 i^{-1}\left\{\left(u_{\bar{z}} c_{z} c^{-1}\right)_{z}-\left(u_{\bar{z}} c_{\bar{z}} c^{-1}\right)_{\bar{z}}\right\} .
$$

Здесъ $S=u_{\bar{z}} \in \mathbb{R}, c=e^{2 \Phi}, \Phi \in \mathbb{R}, \partial_{z}=\partial_{x}-i \partial_{y}, \partial_{\bar{z}}=\partial_{x}+i \partial_{y}$,

$$
c_{t}-4 c_{x y}=8 a_{y} c=-4 \operatorname{Im}\left(u_{z}\right) c, \quad S_{t}+4 S_{x y}=8\left\{S \Phi_{x y}-S_{x} \Phi_{y}-S_{y} \Phi_{x}\right\},
$$

$S=a_{x}-b_{y}, a_{y}+b_{x}=0, u=a+i b$.

Условие $S=0$ дает уравнение $B_{2}$-II:

$$
c_{t}-4 c_{x y}=T(x, y, t) c, \quad \Delta T=0, \quad T \in \mathbb{R}, \quad T=8 a_{y} .
$$

Для самосопряженного факторизованного оператора $L$, у которого $S=0$, кривые $\Gamma^{\prime}, \Gamma^{\prime \prime}$ из теоремы 1 переставляются антиинволюцией $\sigma: \Gamma^{\prime} \rightarrow \Gamma^{\prime \prime}, \Gamma^{\prime \prime} \rightarrow \Gamma^{\prime}, \sigma^{2}=1$. Теория основного состояния таких операторов развивается в [5]. Это - теория чисто магнитного нерелятивистского оператора Паули для частицы со спином 1/2.

\section{Список литературы}

[1] С. В. Манаков, УМН, 31:5 (1976), 245-246. [2] Б. А. Дубровин, И. М. Кричевер, С. П. Новиков, Докл. АН СССР, 229:1 (1976), 15-18. [3] А. П. Веселов, С. П. Новиков, Докл. AH CCCP, 279:4 (1984), 784-788. [4] B. G. Konopelchenko, Inverse Problems, 4:1 (1988), 151-163. [5] P. G. Grinevich, A. E. Mironov, S. P. Novikov, arXiv: 1004.1157.

П. Г. Гриневич (P. G. Grinevich)

Институт теоретической физики им. Л. Д. Ландау РАН

А. Е. Миронов (А. Е. Mironov)

Институт математики им. С. Л. Соболева СО РАН

\section{С. П. Новиков (S. P. Novikov)}

Институт теоретической физики им. Л. Д. Ландау РАН

E-mail: novikov@ipst.umd.edu (University of Maryland)
Представлено В. М. Бухштабером Принято редколлегией 04.05.2010 